\title{
Morphology 'Today'
}

\author{
Markus Müntener*
}

\begin{abstract}
Some aspects of morphological research are presented. Large series of overload experiments of the hind limb have clearly shown that changes in one limb always have consequences for the contralateral non-treated limb. Thus, in such experiments the non-operated limb should never be used as a 'normal' control. Investigations of the extraocular muscles of cats have demonstrated a peculiarity. There was neither utrophin immunoreactivity nor positive staining of the acetylcholine receptors with alpha-bungarotoxin. The studies of the erector trunci muscles in more than 300 low-back pain patients have shown that many conservative therapies are comparable in their outcome with respect to efficiency but not with respect to their costs. Selected historic and recent osseous samples showed a suggested microevolutionary increase for most of the spinal dimensions.
\end{abstract}

Keywords: Extraocular muscles · Low-back pain · Spinal microevolution · Surgical overload

Some aspects of macro- and microscopical research will be presented.

Experimental morphological investigations are generally performed in animals. In rat muscle specific plastic reaction patterns in more phasic and more tonic muscles were studied. Additionally an eventual correlation of the disturbance of locomotion with changes of the fiber pattern was investigated. Fiber size and distribution were analyzed (by histochemistry, immuno-histochemistry, image analysis) in normal and surgically overloaded muscles which had been previously self- or foreign-reinnervated or transplanted. These parameters were analyzed on the operated and on the contralateral side. Both sides were compared with muscles of untreated control animals. These experiments have clearly shown that changes in one limb always have conse- quences for the contralateral non-treated limb. In some animals the reactions were even stronger in the contralateral than in the operated limb. Thus, in such experiments the non-operated limb should never be used as a 'normal' control.

Duchenne muscular dystrophy (DMD) is one of the most common inherited neuromuscular diseases and occurs in about 1:3500 live human male births. The major symptom of DMD patients is the increasing muscle weakness due to the almost complete absence of dystrophin in skeletal muscles. The extraocular muscles (EOMs) remain unaffected during the course of the disease. There are three animal models of DMD: mdx mouse, dog and cat. In cats skeletal and extraocular muscles have been investigated immunohistochemically with respect to the presence of dystrophin and utrophin at the neuromuscular junctions (Fig. 1).

These investigations have demonstrated a peculiarity of the extraocular muscles of the cat. There was neither utrophin immunoreactivity nor positive staining of the acetylcholine receptors with alpha-bungarotoxin.

In humans - in collaboration with clinical institutions - muscles of the shoulder and back were investigated. The muscle biopsies were harvested during surgery or transcutaneously. The majority of the biopsies were harvested from low-back pain patients with a large spectrum of orthopedic symptoms such as e.g. spinal instability (of traumatic, degenerative or inflammatory origin), spinal stenosis or low-back pain. The muscle biopsies were harvested at three time points: a) before surgery, b) postoper-

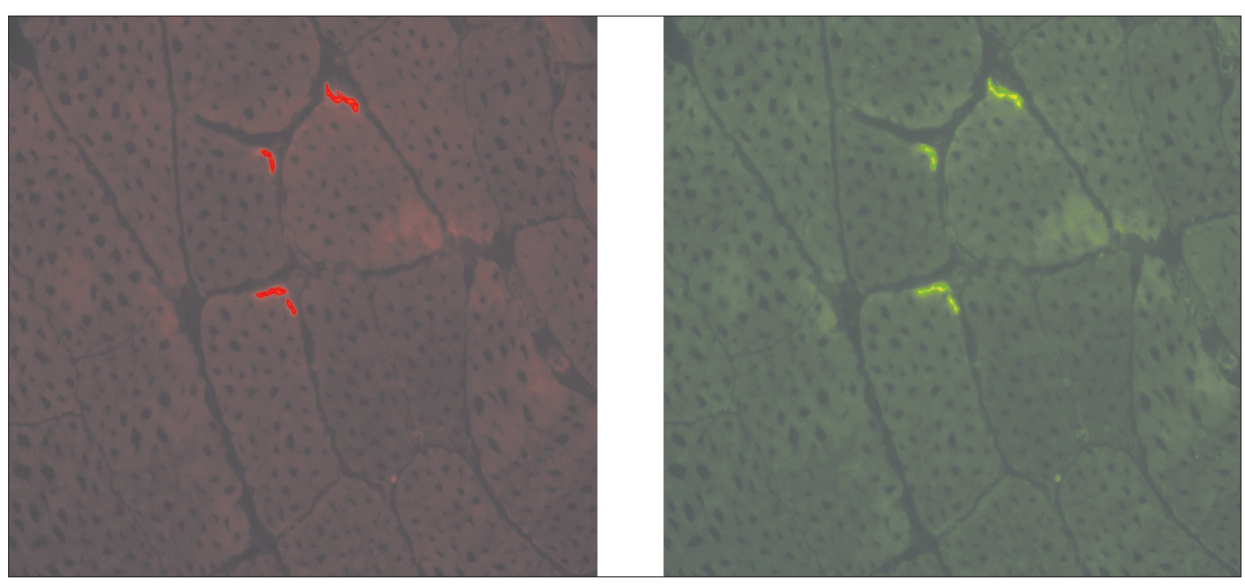

Fig. 1. Consecutive sections of neuromuscular junctions stained for utrophin (red) and alpha-bungarotoxin (green) 


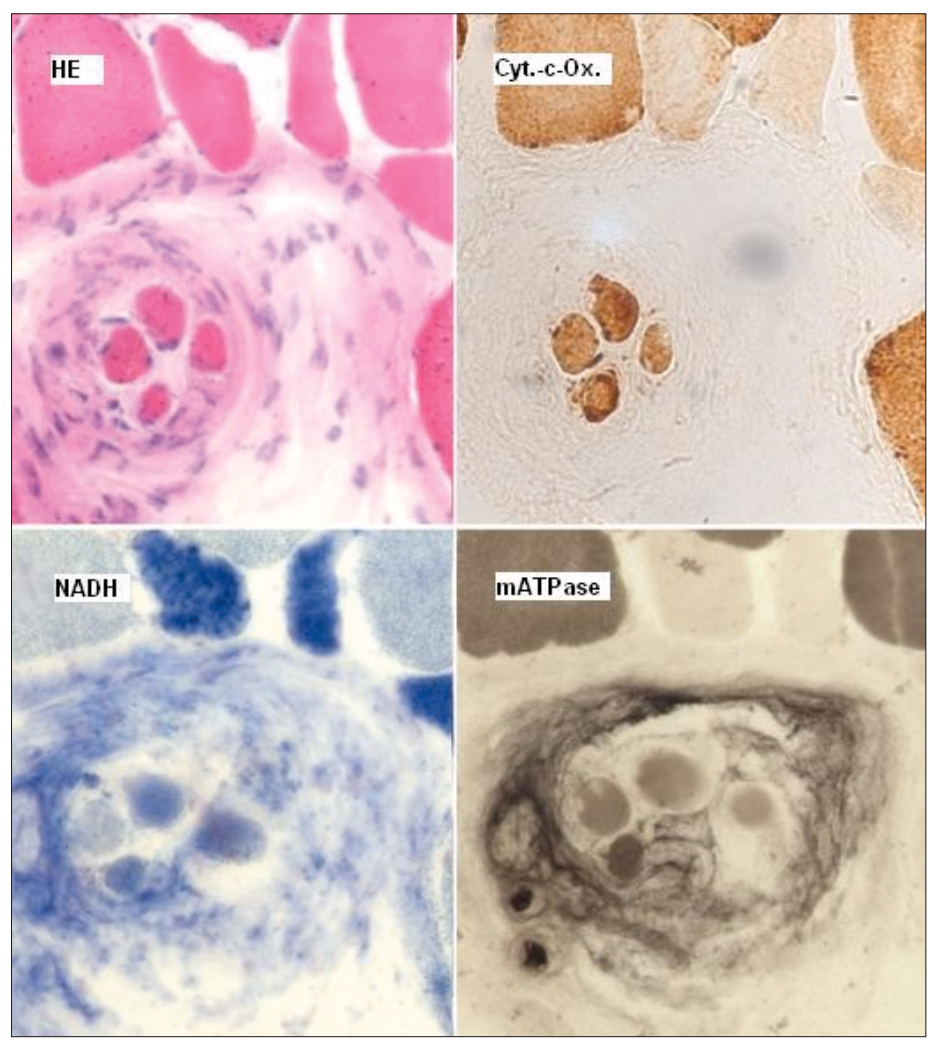

Fig. 2. Consecutive sections of the multifidus muscle of a 'lowback pain' patient. Examples of histochemical reactions as staining for myofibrillar ATPase (mATPase), $\mathrm{NADH}$, cytochrome-coxidase and HE. Muscle fibers surrounding a muscle spindle (a muscle receptor for proprioception).

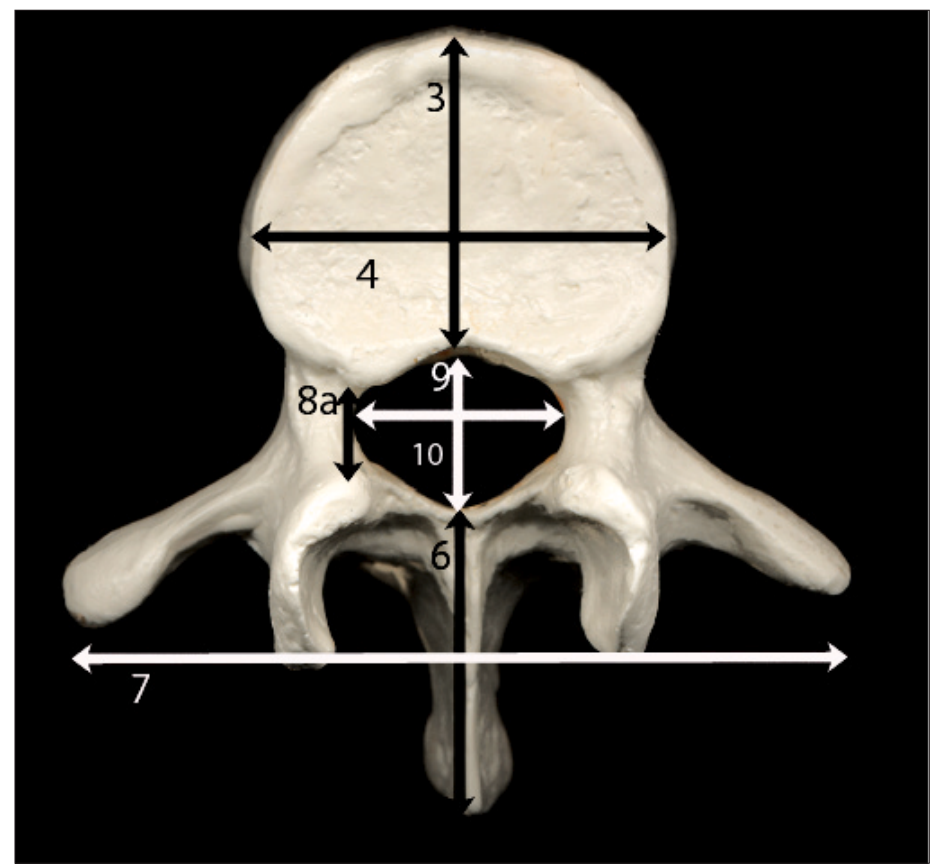

Fig. 3. Human vertebra. Some anthropological measurements are indicated. atively at the start of the postoperative rehabilitation therapy (e.g. physiotherapy, strength therapy, aerobics) and c) at the end of the rehabilitation therapy. In the past decade we have investigated over 1000 muscle biopsies mainly from erector spinae muscle from these patients. The muscles were investigated functionally (EMG, endurance and fatigue resistance), morphologically (magnetic resonance imaging, muscle biopsy, histochemistry) and morphometrically (by image analysis, fiber size and distribution). The morphological analy- sis in particular comprised the histochemical determination of metabolic and functional characteristics of the muscle fibers (Fig. 2), the determination of the fiber types and the analysis of the fiber size and distribution.

These investigations had two goals: the analysis of an eventual correlation of the clinical symptoms with the muscle characteristics and - as follow-up studies after different therapies - the evaluation of the effect of different therapies. The studies have shown that many conservative therapies are comparable in their outcome with respect to efficiency but not with respect to their cost. Additionally in these muscles the gene expression in degenerating or regenerating muscle fibers was studied. The muscles of the patients were compared with corresponding muscles from healthy volunteers in matched (gender and age) groups.

For most parts of the human body, the morphometry and its variation with regard to secular trends, sex dimorphism and individual aging are well known. Surprisingly, research focusing on the vertebral column has so far primarily used either a macroevolutionary or clinically orientated focus. Therefore the osteometry and variation of the human spine was addressed not only in relation to sex and individual age, but also from an additional perspective of possible microevolutionary alterations. Selected historic and recent osseous samples showed a suggested microevolutionary increase for most of the spinal dimensions (Fig. 3), with both mean values and standard deviations rising.

This finding of higher intra-group variability in modern times could be explained as a result of so-called 'relaxed natural selection'. The detected relative smaller size and decrease with age of the bony outline of the neural pathways in males, postulates their higher vulnerability to modern lowback pathologies.

\section{Acknowledgements}

These investigations were mainly performed in collaboration with M. Assadi, J. Dvorak, M. Flück, D. Grob, M. Henneberg, L. Käser, M. Klein, D. Krebs, D.-X. Lu, A.F. Mannion, A. Rhyner, F.J. Rühli, B. Weber, E. Weber, B. Zawadowska. 